NASA Technical Memorandum 102564

\title{
Probabilistic Analysis of Bladed Turbine Disks and the Effect of Mistuning
}

A.R. Shah and V.K. Nagpal

Sverdrup Technology, Inc.

NASA Lewis Research Center Group

Cleveland, Ohio

and

C.C. Chamis

National Aeronautics and Space Administration

Lewis Research Center

Cleveland, Ohio

Prepared for the

31st Structures, Structural Dynamics and Materials Conference cosponsored by the AIAA, ASME, ASCE, AHS, and ASC Long Beach, California, April 2-4, 1990

\section{N/Sก}

$$
\begin{aligned}
& \text { (NACA-TN-10?DGH) PO GOAILISTIL ANALYSIS OF } \\
& \text { NOU-21 } 171
\end{aligned}
$$

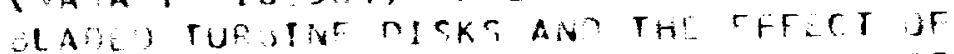

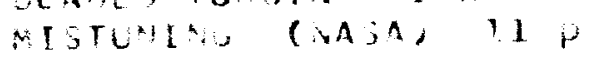

$$
\begin{aligned}
& \text { CDL } ? \angle A
\end{aligned}
$$




\title{
PROBABILISTIC ANALYSIS OF BLADED TURBINE DISKS
}

\author{
AND THE EFFECT OF MISTUNING
}

\author{
A.R. Shah and V.K. Nagpal \\ Sverdrup Technology, Inc. \\ NASA Lewis Research Center Group \\ Cleveland, Ohio 44135 \\ and \\ C.C. Chamis \\ National Aeronautics and Space Administration \\ Lewis Research Center \\ Cleveland, Ohio 44135
}

\section{SUMMARY}

Probabilistic assessment of the maximum blade response of a mistuned rotor (bladed disk assembly) is performed using the computer code NESSUS. The uncertainties in natural frequency, excitation frequency, amplitude of excitdtion and damping have been included to obtain the cumulative distribution function (CDF) of blade responses. Advanced mean value first order analys is is used to compute the CDF. The sensitivities of different random variables are identified. The effect of the number of blades on the mistunuing of rotor is evaluated. It is shown that the uncertainties associated with the forcing function parameters have significant effect on the response distribution of the bladed rotor.

\section{INTRODUCTION}

This paper demonstrates the applicability of the computer code NESSUS (Numerical Evaluation of Stochastic Structures Under Stress) developed at NASA Lewis Research Center (refs. 1 and 2) to obtain probabilistic assessment of a mistuned bladed disk assembly. The inherent uncertaintles in the blade geometry, material properties, damping, mass, etc. result in uncertainties of dynamic characteristics. Due to these uncertainties, each blade on a rotor disk does not have same fundamental frequencies and damping characteristics. These differences are referred to as mistuning. When the blades with such small variations in frequencies interact together on a rotor disk, their individual amplitude and dynamic stresses are altered significantly. This is nothing but the effect of mistuning. The overall disk assembly will have many vibration modes which are very close to each other. The closely spaced modes amplifies the responses significantly. Many researchers have investigated the effect of mistuning both in a deterministic (ref. 3 ) and nondeterministic (probabilistic) (ref. 4) sense. Main focus of those investigations has been on uncertainties in the blade characteristics. However, in reality the uncertainties are also associated with the loads.

This paper includes both structural and load uncertainties. The nondeterministic analysis can be performed by Monte Carlo simulation technique or any other advanced probability integrator method. Monte Carlo simulation 
technique is the most reliable method but it is computationally inefficient and expensive. The probabilistic methods available in NESSUS have been applied to the problem of mistuning and found to be efficient and economical to compute even the low level probability responses. The unique feature of NESSUS is that the reliability analysis can be performed directly for a given statistical distribution of resistance. The outcome of the reliability analysis can be easily and directly used for the design and risk analysis purposes.

\section{NESSUS - BRIEF DESCRIPTION}

NESSUS is a comprehensive Probabilistic Finite Element Analysis computer code to perform static, dynamic, buckling and nonlinear analysis. Various probabilistic and finite element methods are integrated into NESSUS in a modular fashion to solve any general purpose structural analysis problem. Different modules in NESSUS can be used independent of each other. Also, all the modules have been linked to automate the reliability analysis of any structure from beginning to end. Three basic major modules namely; NESSUS/PRE, NESSUS/FEM and/NESSUS/FPI form the core of code. The usage of NESSUS in general involves the identification of primitive variables, their statistical distributions, structural geometry, loads, boundary conditions, etc. Any strength of statistical correlation can be input to NESSUS.

NESSUS/PRE is a preprocessor which allows the user to describe any Gaussian random field over the spatial domain defined in terms of discrete finite element nodes. The Gaussian random field is defined in terms of random variables at nodes with their mean, standard deviation and appropriate form of correlation. Correlated random variables are decomposed into a set of uncorrelated independent vectors using modal analysis by NESSUS/PRE.

NESSUS/FEM is a general purpose finite element code to perform structural analysis and evaluation of sensitivity due to variation in different uncorrelated primitive variables. The response surface defined in terms of random variables required for probabilistic analysis in NESSUS/FPI is obtained discretely in NESSUS/FEM by perturbing independent random variables obtained from NESSUS/PRE. NESSUS/FEM incorporates an efficient perturbation algorithm to compute the sensitivity of random variables. The response sensitivity data generated is stored on a file using linked list database structure. NESSUS/FPI has several advanced rellability methods including fast Monte-Carlo simulation, the most efficient one being the Fast Probability Integrator. This module extracts the data for a given response from the database created by NESSUS/FEM. Since FPI needs explicit response function, the extracted numerical data is used to develop performance model in terms of independent random variables. Using thus developed performance model and statistical distributions of primitive variables, the cumulative distribution is obtained for a given response using fast probability integration.

\section{BLADED DISK ASSEMBLY PROBLEM}

The objective of this paper is to demonstrate the applicability of NESSUS to the problem of a bladed disk assembly as described in the introduction. The aim is: (1) to obtain cumulative probability distribution function (CDF) of the blade amplitudes and (2) to evaluate the effects of: (a) number of 
blades on a disk (b) the statistical distribution of primitive variables and (c) the order of excitation. The following cases are simulated to achieve these objectives:

(1) Disk with 10 blades

(a) First order excitation

(b) Second order excitation

(c) Mean excitation frequency smaller than mean blade frequency

(2) Disk with 20 blades

(a) First order excitation

(b) Second order excitation

\section{(A) Finite Element Model}

The finite element model consists of geometry and loads.

Geometry. - A lumped mass-spring model representing the blades and rotor disk has been used for the purpose of analysis. The disk is represented by lumped masses connected together by a set of radial and circumferential springs. The masses representing the blades are mounted on each disk lumped mass as shown in figure 1. Each blade is modeled by two lumped masses one of which corresponds to the aerofoil region and the other to the shank and fur-tree region. The lumped masses are connected by beam elements. The radial and circumferential beam elements for the disk have only extensional stiffness representing the respective stiffnesses for the disk. The beam elements connecting blade masses have only bending stiffness to represent the flexural mode of vibration of the blade. Therefore, it is important to note that only the bending blade mode of vibration is considered in the analysis since it normally dominates the responses. Thus, the dynamic interaction between blades comes through disk springs and masses.

Loads. - The force induced on blades is due to variations in the flow around circumferential direction. The variations in the flow are considered to be periodic in nature. Therefore, the excitation would be harmonic which is characterized by amplitude, frequency of excitation and phase angle. The magnitude of amplitude does not matter in case of linear analysis, however its statistical distribution does since the assessment is made on the responses normalized to those of tuned rotor. The frequency of excitation is selected as integer multiple of engine rpm. Since, the excitation is harmonic in nature, it differs from blade to blade in terms of phase angle only. As the blades are fixed around the circumference, the phase difference, $\phi$ between blade's excitation is given by

$$
\phi=\frac{2 M \pi}{N}
$$

where $M$ is the integer multiple between engine rpm and frequency of excitation

$\mathrm{N}$ is the number of blades on a disk 
The modal damping used in the analys is is 3 percent (includes material, structural and aerodynamic).

\section{(B) PROBABILISTIC MODEL}

The following variables are considered to be random in the analysis:

(1) Frequency of blade

(2) Damping

(3) Frequency of excitation

(4) Amplitude of excitation

The frequency of the individual blade depends on its geometry, material property and the mass. Since frequency is not a primitive variable input to NESSUS, modulus of elasticity, $E$ of blade is substituted for it as a random variable. This is done in such a way that the mean value of $E$ gives mean frequency and one standard deviation (SD) of $E$ from its mean gives frequency equal to one SD from its mean. In this simulation the uncertainties in the geometry and the mass are represented by equivalent uncertain moduli. Normally, the rotor disk is balanced by placing blades of the same moment weight in opposite slots. To include such a physical requirement in a probabilistic model, it is assumed that the blades in opposite slots are fully correlated statistically. The mean, standard deviation and distributions of different random variables are given in table I.

Since, NESSUS/FPI requires a performance model (response surface) of response to compute the CDF, the sensitivity of each random variable is obtained by perturbing them one at a time. Each blade frequency (modulus of elasticity) is considered to be an independent random variable in the analysis to develop a suitable performance model of mistuning.

\section{(C) MATHEMATICAL ALGORITHM DISCUSSION}

The governing equation of motion for the bladed disk is given by

$$
[M]\{\ddot{X}\}+[C]\{X\}+[K]\{X\}=\left\{A e^{i(\omega t-\phi)}\right\}
$$

where $\ddot{x}, \dot{x}, x$ is acceleration, velocity and displacement respectively

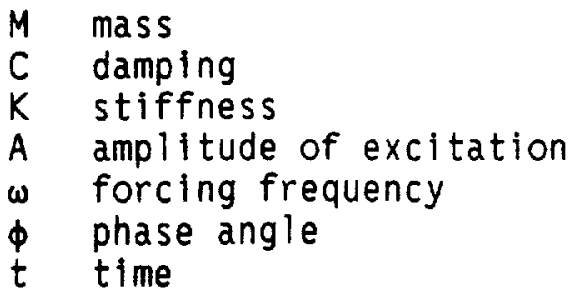

NESSUS uses the modal response transfer function approach to calculate the modal responses. Modal responses are superposed to obtain total response. 
One of the major problems encountered in the analysis of mistuned rotors is to extract fairly large number of closely spaced eigenvalues. There are at least as many closely spaced frequencles as the number of blades. Also, the fact that the first blade on a disk is connected to the $N$ th blade, there exists a nonzero term in the corner of stiffness matrix. Thus the stiffness matrix becomes sparse rather than banded. NESSUS has a powerful profile storage scheme and a subspace Iteration eigenvalue extraction algorithm. When the number of closely spaced modes increase, the tolerance provided for iterative procedure should be small. The algorithm did not show any sign of numerical instability and the eigenvalues were extracted in relatively few iterations. The other salient feature of the algorithm, in conjunction with perturbation procedure, is that the eigenvalues and eigenvectors of the previous perturbation are used as initial trial vector for the current perturbation in order to obtain rapid convergence and increased accuracy.

Advanced mean value first order analysis (AMVFO) (ref. 5) has been used to obtain CDF of amplitudes. The procedure involved can be summarized as follows:

(1) Obtain the linear approximation of response, $z_{1}(X)$ based on perturbation about mean value.

(2) Compute values of independent variables, $Z^{*}$ for selected CDF values (mean value first order analysis).

(3) Recompute $z\left(\bar{X}^{*}\right)$ to correct the response for the same CDF in step (2) (Advanced mean value first order analysis).

It has been found that the AMVFO gives very accurate results efficiently.

\section{DISCUSSION OF RESULTS}

Cumulative probability distribution of normalized amplitude of the maximum blade response on a disk are plotted. The normalized amplitude is defined as the ratio of maximum blade response of a mistuned rotor to that of the tuned rotor. The tuned rotor is the one for which all the individual blade frequencies are the same. The normalized response indicates the amplification of response due to mistuning. Figures 2 and 3 depict the CDF of normalized blade amplitude for a rotor with 20 blades and figures 4,5 and 6 for the rotor with 10 blades. As expected, the results show that for the same probability level for a given rotor, the amplification is higher when the excitation frequency is closer to the natural frequency of a blade. Also, it is observed that for a given order of excitation, the amplification increases as the number of blades increase. This is due to the fact that as the number of blades increase, a higher number of vibration modes get closer to each other. The following is very interesting and worth noting (refer to table II):

(1) When mean excitation frequency is larger than the mean blade natural frequency, the uncertainties in the excitation frequency are more sensitive to response CDF than those of blade frequency. 
(2) When mean excitation frequency is smaller than the mean blade natural frequency, the uncertainties of blade frequency become significantly sensitive to response CDF as compared to those of excitation frequency.

The sensitivity of the frequency of a blade or the frequency of excitation depends largely on the difference between the mean of each frequencies and their coefficient of variations. If:

(1) The coefficient of variation for the blade frequency is small compared to that of the excitation frequency.

(2) The means of the two frequencies are fairly far apart.

(3) The mean excitation frequency is larger than mean blade frequency.

Then it is obvious that the uncertainty in the excitation frequency will dominate the response distribution of the rotor. The point to note is that it is very important to consider uncertainties in the blade frequency and excitation forces simultaneously in order to properly assess their respective sensitivities on the response of the rotor.

\section{CONCLUDING REMARKS}

It is demonstrated that the uncertainties of mistuned bladed disks can be evaluated using NESSUS. Structural and modeling considerations related to the problem have been included. The effect of the number of blades, and the order of excitation have been identified. The specific findings are as

follows:

(1) When the mean excitation frequency is larger than the mean blade frequency:

The effects of uncertainties in the excitation frequency to mistuning is found to be very significant as compared to the effects of uncertainties in the blade frequency. Therefore, it is very important to simultaneously conslder both structural and load uncertainties in the analysis.

(2) When the mean excitation frequency is smaller than the mean blade frequency:

The effects of uncertainties in the blade frequency are more significant in comparison to those of excitation frequency.

These findings, (1) and (2), and corresponding sensitivities help designers achleve robust rotor designs. For example, when the scatter in the blade frequency is larger and uncontrollable, then the rotor should be designed to operate beyond resonance and vice versa. The blade can be designed for a desired probability level and its corresponding amplification of response. Also, the sensitivities provide directions (through the primitive variables) to enhance quality control in order to reduce the uncertainty in the rotor response and consequently the risk. 


\section{REFERENCES}

1. Chamis, C.C.: Probabilistic Structural Analysis Methods for Space Propulsion System Components. NASA TM-88861, 1986.

2. Probabilistic Structural Analysis Methods (PSAM) for Select Space Propulsion Components, User's Manual, Nov. 1989.

3. Dye, R.C.F.; and Henry, T.A.: Vibration Amplitudes of Compressor Blades Resulting From Scatter in Blade Natural Frequencies. J. Eng. Power, vol. 91, no. 3, July 1969, pp. 182-188.

4. Griffin, J.H.; and HoosaC, T.M.: Model Development and Statistical Investigation of Turbine Blade Mistuning. J. Vibr. Acoust. Stress Rel. Des., vol. 106, no. 2, Apr. 1984, pp. 204-210.

5. Wu, Y.T.; and Burnside, O.H.: Efficient Probabilistic Structural Analysis Using an Advanced Mean Value Method. Probabilistic Methods in Civil Engineering, P.D. Spanos, ed., American Society of Civil Engineers, New York, 1988, pp. 492-495. 
TABLE I. -

[Statistical distribution of all primitaive variables considered

is normal.]

(a) Disk with 10 blades.

\begin{tabular}{|c|c|c|}
\hline Primitive variable & Mean & $\begin{array}{c}\text { Coefficient of } \\
\text { variation, } \\
\text { percent }\end{array}$ \\
\hline $\begin{array}{l}\text { Frequency of blade (cps) } \\
\text { Damping } \\
\text { (a) First order excitation } \\
\text { Frequency (cps) } \\
\text { Amplitude } \\
\text { (b) Second order excitation } \\
\text { Frequency (cps) } \\
\text { Amplitude } \\
\text { (c) Mean excitation frequency } \\
\text { smaller than mean blade } \\
\text { frequency } \\
\text { Frequency (cps) } \\
\text { Amplitude }\end{array}$ & $\begin{array}{l}959.0 \\
3 \text { percent } \\
1909.86 \\
100.0 \\
2864.8 \\
100.0\end{array}$ & $\begin{array}{l}1.0 \\
5.0 \\
5.0 \\
5.0 \\
5.0 \\
5.0\end{array}$ \\
\hline
\end{tabular}

(b) Disk with 20 blades.

\begin{tabular}{|c|c|c|}
\hline Primitive variable & Mean & $\begin{array}{l}\text { Coefficient of } \\
\text { variation, } \\
\text { percent }\end{array}$ \\
\hline $\begin{array}{l}\text { First of blade (cps) } \\
\text { Damping } \\
\text { (a) First order excitation } \\
\text { Frequency (cps) } \\
\text { Aplitude } \\
\text { (b) Second order excitation } \\
\text { Frequency (cps) } \\
\text { Amplitude }\end{array}$ & $\begin{array}{l}959.0 \\
3 \text { percent } \\
1909.86 \\
100.0 \\
2864.8 \\
100.0\end{array}$ & $\begin{array}{l}1.0 \\
5.0 \\
5.0 \\
5.0 \\
5.0 \\
5.0\end{array}$ \\
\hline
\end{tabular}

TABLE II. - SENSITIVITY FACTORS

[Case (a), (b), (c) etc. refer to those of table I.]

(a) Disk with 10 blades.

\begin{tabular}{|c|l|c|}
\hline Probability & Primative Variable & Sensitivity \\
\hline (a) 0.99999 & Blade frequency & 0.15 \\
& Excitation frequency & .916 \\
(b) .99999 & Blade frequency & .017 \\
& Excitation frequency & .953 \\
(c) .999999 & Blade frequency & .527 \\
& Excitation frequency & .657 \\
\hline
\end{tabular}

(b) Disk with 20 blades.

\begin{tabular}{|l|l|c|}
\hline Probability & Primative Variable & Sensitivity \\
\hline (a) 0.99999 & Blade frequency & 0.124 \\
& Excitation frequency & .915 \\
(b) .99999 & Blade frequency & .051 \\
& Excitation frequency & .91 \\
\hline
\end{tabular}




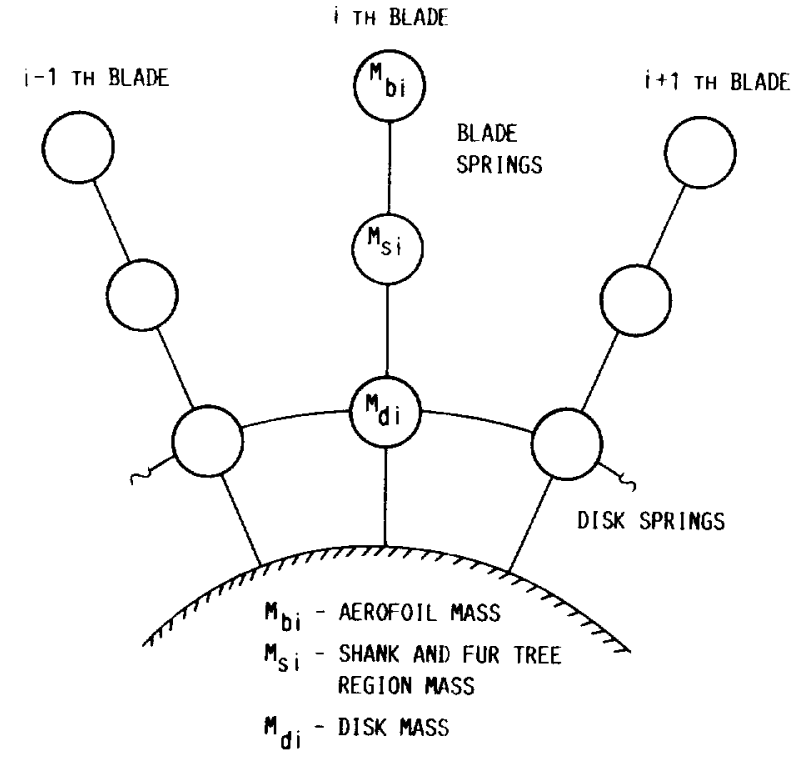

FIGURE 1. - LUMPED MASS-SPRING MODEL. FOR BLADED DISK.

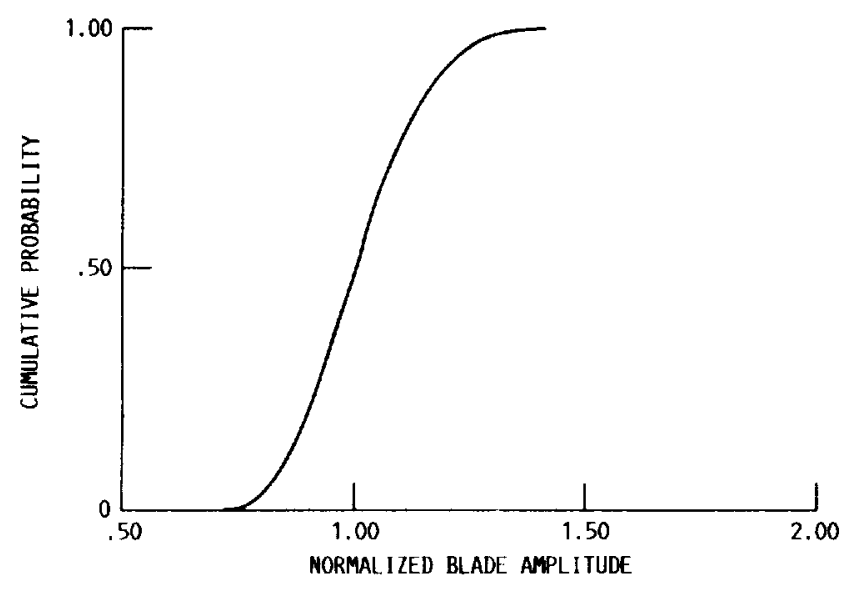

FIGURE 3. - MISTUNING OF BLADED DISKS, 20 BLADES 3RD ORDER EXCITATION.

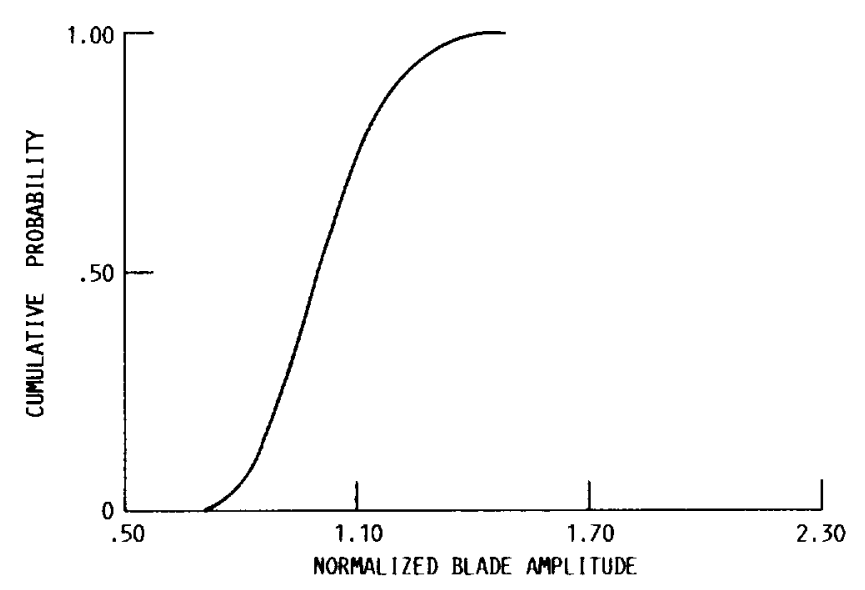

FIGURE 2. - MISTUNING OF BLADED DISKS. 20 BLADES 2ND ORDER EXCITATION.

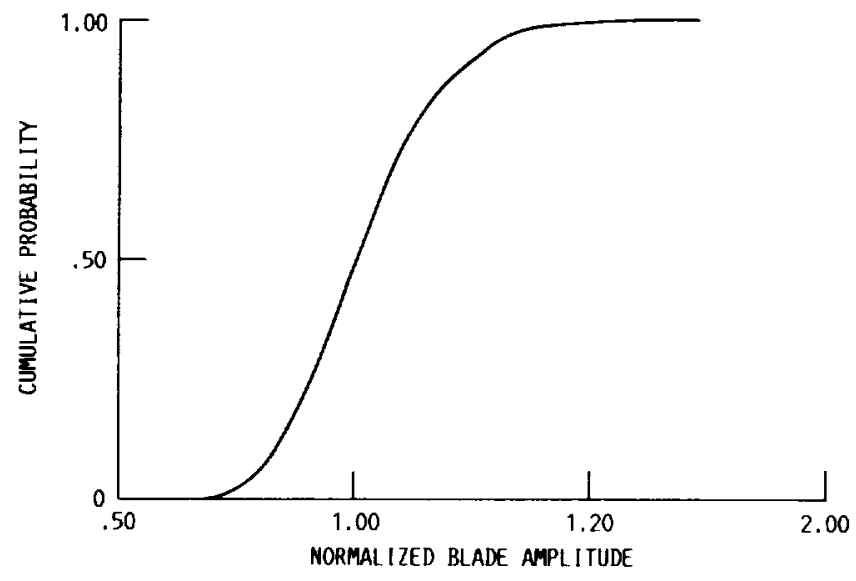

FIGURE 4. - MISTUNING OF BLADED DISKS. 10 BLADES 2ND ORDER EXCITATION.

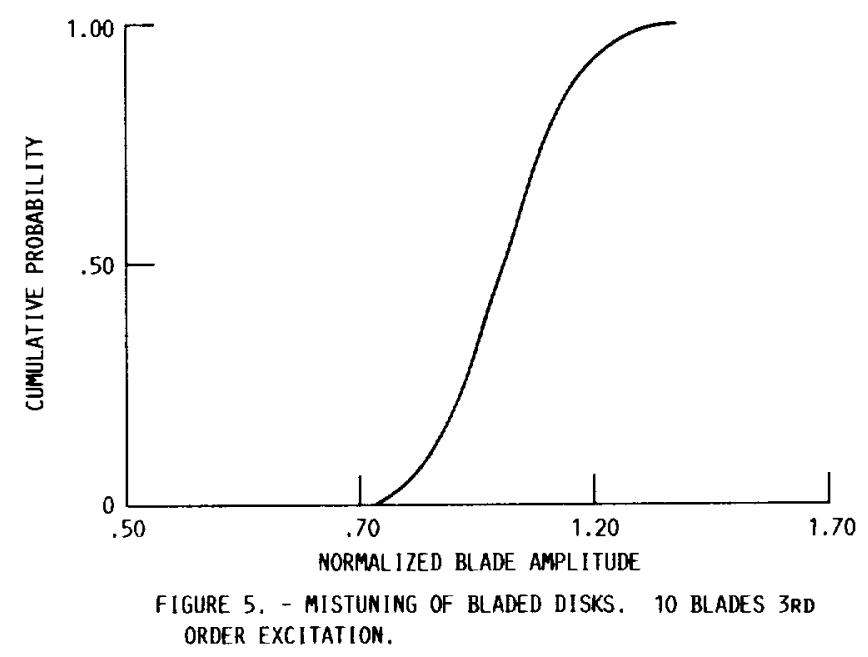




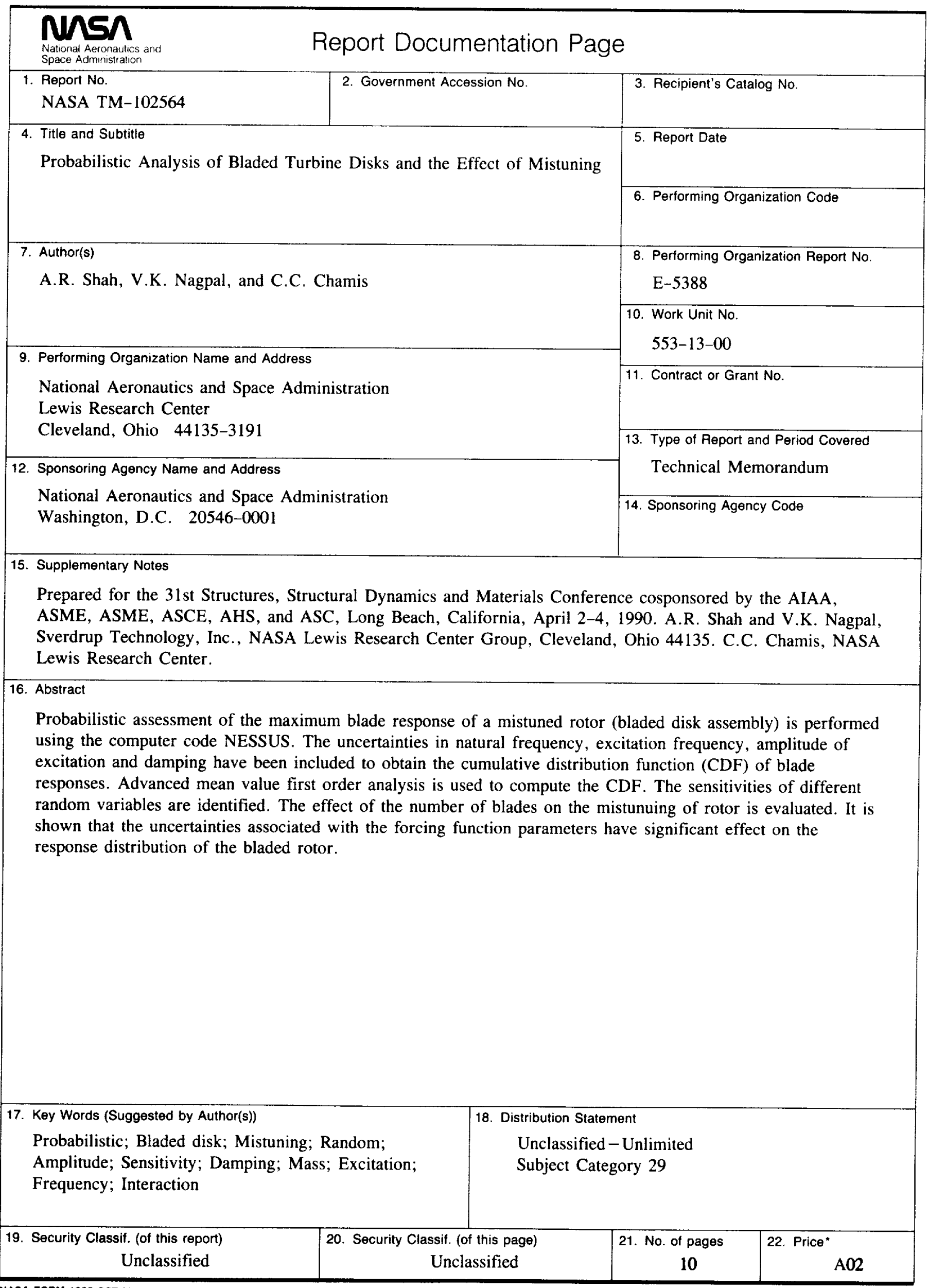

